\title{
Stimuli-Responsive Polymers in Nanotechnology: Deposition and Possible Effect on Drug Release
}

\author{
A.L. Yarin ${ }^{1}$ \\ Department of Mechanical and Industrial Engineering, University of Illinois at Chicago \\ Chicago, IL 60607-7022, USA
}

\begin{abstract}
Stimuli-responsive polymers result in on-demand regulation of properties and functioning of various nanoscale systems. In particular, they allow stimuli-responsive control of flow rates through membranes and nanofluidic devices with submicron channel sizes. They also allow regulation of drug release from nanoparticles and nanofibers in response to temperature or $\mathrm{pH}$ variation in the surrounding medium. In the present work two relevant mathematical models are introduced to address precipitation-driven deposition of surface layers of stimuli-responsive polymers and describe a possible effect of swelling-shrinkage transition of such polymers on drug release.
\end{abstract}

Key words: polymer deposition, stimuli-responsive polymers, drug release, fluid and solid mechanics, probability theory

AMS subject classification: 76R05, 74R10, 60K40

\section{Introduction}

The most popular stimuli-responsive polymer explored and used in various applications is Poly (N-isopropyl acrylamide) (PNIPAM). PNIPAM is a temperature- and $\mathrm{pH}$-responsive polymer. Namely, at temperatures below $32{ }^{\circ} \mathrm{C}$ PNIPAM swells, while at temperatures above $32{ }^{0} \mathrm{C}$ it shrinks in aqueous environment. Moreover, co-polymers of PNIPAM allow for a wide variation of the swelling/shrinkage temperature. Similar swelling-shrinkage transitions of stimuli-responsive polymers happen in response to variation in $\mathrm{pH}$, ionic strength, solvent composition and magnetic field. Blends of PNIPAM with such biocompatible and biodegradable polymers as Poly(acrylic acid) (PAA) can be easily prepared, which yield PAA polymer matrixes with PNIPAM islands

\footnotetext{
${ }^{1}$ E-mail: ayarin@uic.edu
} 
inside. Electrospinning, co-electrospinning and various solution-precipitation methods allow for manufacturing of nanofibers and nanoparticles with embedded or grafted macromolecules [1-5]. In addition, nanofibers and nanoparticles functionalized with drugs attract significant interest in biomedical applications [6], where PNIPAM-like stimuli-responsive materials embedded in the bulk or grafted at the surface may facilitate efficient drug delivery systems.

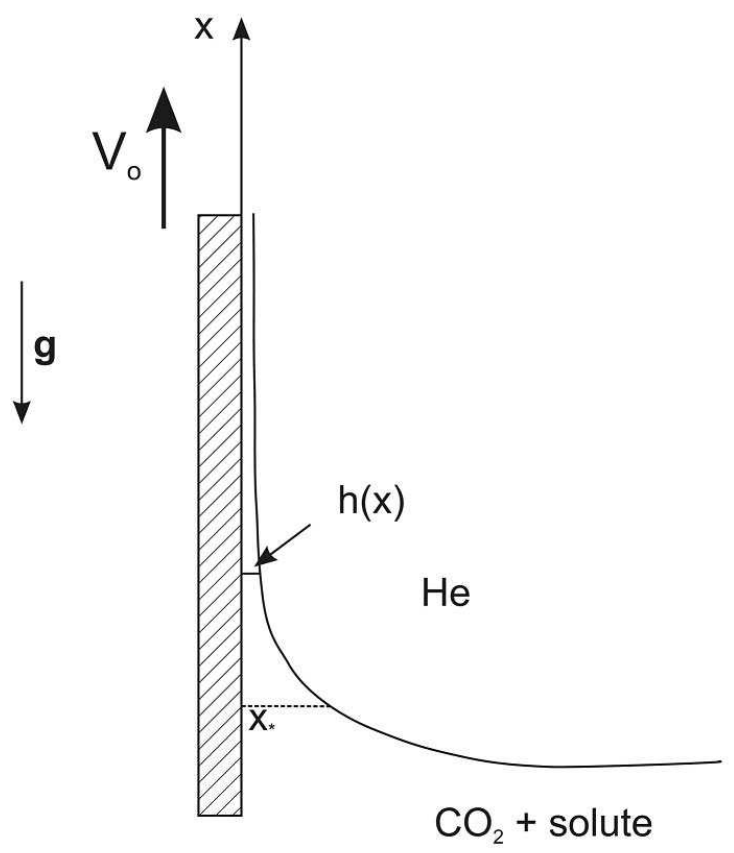

Figure 1: Sketch of the deposition process.

PNIPAM is available in a range of molecular weights from about $10 \mathrm{kDa}$ to $100 \mathrm{kDa}$. Therefore, its macromolecules are typically relatively small. Direct deposition of thin PNIPAM layers on solid surfaces is frequently desirable. Deposition of particles nucleated from small organic molecules dissolved in supercritical liquid carbon dioxide displaced by supercritical liquid helium was studied experimentally in [7], whereas deposition from evaporating layers of supercritical liquid carbon dioxide was studied in [8]. In particular, a potential deposition mechanism in the displacement experiment was recognized (sketched in Fig. 5 in [7]; cf. Fig. 1 in this work). Namely, supercritical liquid carbon dioxide containing dissolved organic molecules is displaced near a vertical wall by a plug flow of non-solvent, supercritical liquid helium, moving downward with velocity $V_{0}$. Due to the no-slip condition at the wall, the interface between the solution and non-solvent acquires a meniscus-like shape with a thin solution layer attached to the wall. In the coordinate frame associated with the displacement front, the situation can be considered as a withdrawal problem, with the wall moving upward with velocity $V_{0}$ and withdrawing an adjacent solution layer. Mutual diffusion of carbon dioxide and helium is very weak. However, it is felt in a thin near-wall layer due to presence of high concentration gradients there. Therefore, the solvent (supercritical liquid carbon dioxide) diffuses from the layer to the bulk of the surrounding non-solvent (supercritical liquid helium), whereas the latter diffuses into the near-wall layer. Both diffusion processes reduce 
solvent quality and, as a result, nucleation and growth of solute particles begins in the bulk of the near-wall layer. These particles begin to deposit onto the wall as a very thin sublayer. It is of interest to predict thickness, uniformity and roughness of deposited sublayer, the size of deposited solute particles and deposition rate. Some of these goals are addressed in Sections 2 and 3 of this work.

Release of model compounds (fluorescent dyes) or drugs from polymer nanofibers with embedded islands of stimuli-responsive material is addressed in Section 4. According to [9], dye and drug release from nanofibers is strongly affected by their nanoporosity, and in fact, only the admixture embedded at nanopore surfaces could be released. Therefore, swelling or shrinkage of PAA (or any other polymer)-PNIPAM nanofibers below or above $32{ }^{\circ} \mathrm{C}$, respectively, can be used to affect drug/dye release on demand. This capability might be of interest in medical applications where tumors are known to have temperatures which differ from the surrounding tissue temperature. More than that, in such applications temperature might be controlled locally. In Section 4 the effect of swelling or shrinkage of the embedded islands on drug/dye release from nanofibers is evaluated. Concluding statements are given in Section 5.

\section{Deposition process: theoretical model}

In the reference frame associated with the displacement front in experiments [7], the hydrodynamical setting resembles the classical withdrawal problem of Landau and Levich [10,11] (cf. Fig. 1). The only difference is the presence of non-solvent (supercritical liquid helium) above the solution meniscus and outside the near-wall solution layer at the moving wall. However, the velocities involved (of the order of $10^{-3}-10^{-2} \mathrm{~cm} / \mathrm{s}$ ) and liquid viscosities (less than that of water) make any dynamic effects of supercritical liquid helium highly improbable. Then, its presence practically cannot affect the flow field in the solution domain. Therefore, it is possible to assume that the flow field corresponding to the classical withdrawal problem holds here as well. In the present work the hydrodynamic solution of Landau and Levich $[10,11]$ is used in the following approximate dimensionless form

$$
u=1+1.89 \exp \left(X_{*}-X\right)\left(\psi^{2} / 2-\psi\right),
$$

where $u$ is the longitudinal fluid velocity in the layer rendered dimensionless by $V_{0} ; X>X_{*}$ is the longitudinal coordinate along the wall rendered dimensionless by $Q(\sigma / 3 \mu)^{1 / 3} / V_{0}^{4 / 3}$ with $Q$ being the flow rate in the near-wall layer, $\sigma$ the interfacial tension between supercritical liquid carbon dioxide and liquid helium, $\mu$ viscosity of carbon dioxide; $X_{*}$ corresponds to the matching of the near-wall layer with the static meniscus, and according to $[10,11]$ is given by $X_{*}=2.193 / \mathrm{Ca}^{1 / 3}$, where $C a=V_{0} \mu / \sigma$ is the capillary number; $\psi$ is the stream function rendered dimensionless by $Q(0 \leq \psi \leq 1)$. Note, that according to $[10,11], Q=0.93[\sigma /(\rho g)]^{1 / 2} \mathrm{Ca}^{2 / 3}$, where $g$ is gravity acceleration. The flow field (2.1) was obtained using the fact that in $[10,11]$ approximately

$$
u=1-3(1-H)\left(Y^{2} / 2-Y\right)
$$




$$
H=1+0.63 \exp \left(X_{*}-X\right)
$$

where the lateral coordinate $Y$ and the thickness of the near-wall layer $H$ are rendered dimensionless by $Q / V_{0}$; also, in the same approximation $\psi=Y$. The second approximation of the relation between $Y$ and $\psi$ is given by the following expression: $Y=\int_{0}^{\psi} u^{-1} d \psi$.

In the near-wall layer the following four species are transferred by convection-diffusion mechanism: (i) carbon dioxide, (ii) helium, (iii) solute molecules and (iv) nucleated and grown solute particles. Their dimensionless number concentrations will be denoted as $C_{1}, C_{2}, C_{s}$ and $C_{p}$, respectively. These concentrations are rendered dimensionless by the initial cumulative (carbon dioxide and solute) number of molecules $C_{0}$ in the solution before it comes in contact with helium. Concentrations $C_{1}, C_{2}, C_{s}$ and $C_{p}$ are governed by the corresponding convection-diffusion equations. It is convenient to present them using the von Mises coordinates $X$ and $\psi$ (to have a rectangular integration domain), as is customary in the boundary layer theory [12]. Then, the dimensionless equations take the form

$$
\begin{gathered}
\frac{\partial C_{i}}{\partial X}=\frac{D_{i}}{(3 C a)^{1 / 3}} \frac{\partial}{\partial \psi}\left(u \frac{\partial C_{i}}{\partial \psi}\right), i=1,2, \\
\frac{\partial C_{s}}{\partial X}=\frac{D_{s}}{(3 C a)^{1 / 3}} \frac{\partial}{\partial \psi}\left(u \frac{\partial C_{s}}{\partial \psi}\right)-\frac{m M}{(3 C a)^{1 / 3}} \frac{J}{u}, \\
\frac{\partial C_{p}}{\partial X}=\frac{D_{p}}{(3 C a)^{1 / 3}} \frac{\partial}{\partial \psi}\left(u \frac{\partial C_{p}}{\partial \psi}\right)+\frac{M}{(3 C a)^{1 / 3}} \frac{J}{u} .
\end{gathered}
$$

In Eqs. (2.4)-(2.6) the species diffusion coefficients $D_{i}(i=1,2), D_{s}$ and $D_{p}$ are rendered dimensionless by $Q ; m$ is an average number of solute molecules comprising a nucleated and grown particle, the dimensionless nucleation rate $J$ is given by Zeldovich theory of nucleation [13-16]:

$$
J=C_{s}^{2} \exp \left(-\frac{E}{(\log S)^{2}}\right)
$$

The nucleation rate is rendered dimensionless by the following scale:

$$
J_{0}=\frac{\gamma_{e f f}^{1 / 2}(2 v)^{2}(1-\cos \theta) C_{0}^{2} \nu \lambda \exp (-\Delta / k T)}{2 V_{p}(\phi(\theta) k T)^{1 / 2}},
$$

where $\gamma_{e f f}$ is the effective interfacial energy of the nucleation process, $v$ is the molecular volume, $\theta$ is the contact angle between the nuclei and the substrate (if they are in contact), $\nu$ is the frequency factor, $\lambda$ the mean free path of particles in the liquid (of the order of the molecular diameter), $\Delta U$ is a desolvation energy, $k$ is Boltzmann's constant, $T$ temperature, $V_{p}$ is the particle volume, $\phi(\theta)=$ $(1-\cos \theta)^{2}(2+\cos \theta) / 4$ is the wetting factor. Also, the dimensionless activation energy $E$ and the ratio $S$ determining the difference in chemical potentials between the solute in the supersaturation solution phase and the solute in the solid phase are given by the following expressions: 


$$
\begin{gathered}
E=\frac{16 \pi}{3} \frac{\gamma_{e f f}^{3} v^{2} \phi(\theta)}{(k T)^{3}}, \\
S=\frac{C_{1, e q}}{C_{1}},
\end{gathered}
$$

where $C_{1, e q}$ is the carbon dioxide concentration in solution in equilibrium with the nucleated solid phase; the equilibrium concentration of solute is $C_{s, e q}=1-C_{1, e q}$, respectively. Also, $M=$ $Q J_{0} /\left(C_{0} V_{0}^{2}\right)$.

In Eqs. (2.4)-(2.6) the longitudinal velocity u is given by Eq. (2.1). These equations are solved with the following initial and boundary conditions:

$$
\begin{gathered}
X=X_{*}:\left\{\begin{array}{c}
0 \leq \psi<1: C_{1}=C_{1, e q}=1-C_{s, e q} ; C_{2}=0 ; C_{s}=C_{s, e q} ; C_{p}=0 \\
\psi=1: C_{1}=0 ; C_{2}=1 ; C_{s}=0 ; \partial C_{p} / \partial \psi=0
\end{array},\right. \\
X>X_{*}:\left\{\begin{array}{c}
\psi=0: \partial C_{1} / \partial \psi=\partial C_{2} / \partial \psi=\partial C_{s} / \partial \psi=0 ; C_{p}=0 \\
\psi=1: C_{1}=0 ; C_{2}=1 ; C_{s}=0 ; \partial C_{p} / \partial \psi=0
\end{array}\right.
\end{gathered}
$$

When a concentration derivative by $\psi$ is zero, it means that the corresponding species flux in lateral direction is zero.

The particle flux to the wall $j_{p}$ rendered dimensionless by $V_{0} C_{0}$ is given by

$$
j_{p}=-\left.D_{p} u \frac{\partial C_{p}}{\partial \psi}\right|_{\psi=0} .
$$

Then, the corresponding distribution of the deposit thickness at the wall $h(X)$ rendered dimensionless by $V_{p} C_{0} Q / V_{0}$ can be found as

$$
h(X)=\frac{1}{(3 C a)^{1 / 3}} \int_{X_{*}}^{X}\left|j_{p}\right| d X .
$$

In the case of particle deposition from a withdrawn layer of supercritical liquid carbon dioxide evaporating from the free surface in a channel of width $R$, it is possible to show using the approximate conformal mapping technique [17] that the dimensionless mass flux of solvent at the free surface $j_{v}$ is given by

$$
j_{v}=\frac{D_{v} C_{v s}}{(R-1)\left(1-\delta(X)+(\pi / 4) p \cdot v \cdot \int_{-\infty}^{\infty}(\delta(\xi)-\delta(X)) / \sinh ^{2} \frac{\pi(\xi-X)}{2} d \xi\right)}
$$

where $j_{v}$ is rendered dimensionless by $V_{0} C_{0}, R-$ by $Q / V_{0}$, the diffusion coefficient of vapor $D_{v}$ by $Q, \xi$ is a dummy variable, and approximately

$$
\delta(X)=\frac{0.63}{R-1} \exp \left(-\left|X-X_{*}\right|\right) .
$$


The integral in the denominator in Eq. (2.15) is evaluated in the principal value sense.

In the case of carbon dioxide evaporating from the free surface in a channel of width $R$, Eqs. (2.4) with $i=1,(2.5)$ and (2.6) are solved with the following initial and boundary conditions

$$
\begin{gathered}
X=X_{*}:\left\{\begin{array}{c}
0 \leq \psi<1: C_{1}=C_{1, e q}=1-C_{s, e q} ; C_{s}=C_{s, e q} ; C_{p}=0 \\
\psi=1:-D_{1} u \partial C_{1} / \partial \psi=j_{v} ; \partial C_{s} / \partial \psi=\partial C_{p} / \partial \psi=0
\end{array}\right. \\
X>X_{*}:\left\{\begin{array}{c}
\psi=0: \partial C_{1} / \partial \psi=\partial C_{s} / \partial \psi=0 ; C_{p}=0 \\
\psi=1:-D_{1} u \partial C_{1} / \partial \psi=j_{v} ; \partial C_{s} / \partial \psi=\partial C_{p} / \partial \psi=0
\end{array}\right.
\end{gathered}
$$

Equations (2.4)-(2.6) with u given by Eq. (2.1) subjected to the conditions (2.11) and (2.12) (or (2.17) and (2.18)) are solved numerically using the method of straight lines, which was previously successfully applied to kindred problems arising in the theory of single- and multiphase jets and torches described in the boundary layer approximation [18-25].

\section{Deposition process: results and discussion}

Solutions of Eqs. (2.4)-(2.6) with u given by Eq. (2.1) subjected to the conditions (2.11) and (2.12) were obtained numerically with the following values of the dimensionless parameters and groups involved: $D_{1}=D_{2}=D_{s}=D_{p}=1, E=10, C_{s, e q}=0.1, m=30, M=10^{4}$, and $C a=0.01$. The values of the numerous physical parameters incorporated in the dimensionless groups relevant for deposition processes can be found in [7]. The corresponding results are presented in Figs. 24. In particular, Fig. 2 shows the solvent concentration field in the near-wall layer. In the layer part attached to the meniscus domain (on the left in Fig. 2) there is a significant concentration of solvent (light area-yellow area online). However, due to diffusion of solvent into the surrounding non-solvent area (above the upper boundary of the near-wall domain shown in Fig. 2), as well as diffusion of non-solvent into the near-wall layer, concentration $C_{1}$ decreases. Then gradually a dark area-blue area online- (low values of $C_{1}$ ) replaces the light area-yellow area online- to the right in Fig. 2.

A decrease in solvent concentration accompanied by an increase in non-solvent concentration in the near-wall layer triggers solute nucleation and particle formation in the layer bulk. The particle concentration has maximal values inside the layer (the light area- yellow area online- in Fig. 3). As a result, there is a particle mass flux toward the wall, which leads to particle layer deposition. The corresponding predicted thickness of the deposited layer is depicted in Fig. 4. It is seen that in the present case particle deposition takes place only at the part of the layer attached to the meniscus domain, namely approximately in the range $10.25 \leq X \leq 11$. In this range the deposited layer thickness increases non-monotonously and non-uniformities should be expected.

A decrease in the average number of solute molecules per grown particle ( $m=3$ instead of $m=30$ ) with the other parameters being fixed obviously does not affect the concentration field of solvent. However, the particle concentration field and the deposited layer thickness along the wall definitely change (Figs. 5 and 6, respectively). In particular, particle concentration increases (more particles are nucleated in a unit volume when more solute molecules are available), as well 


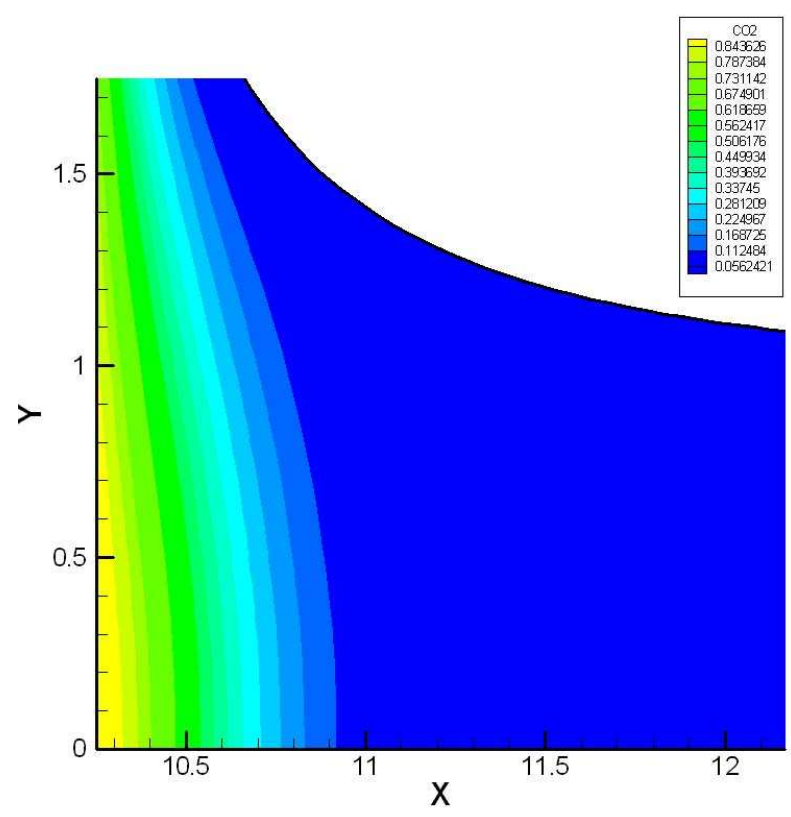

Figure 2: (Color online.) Solvent concentration field in the near-wall layer.

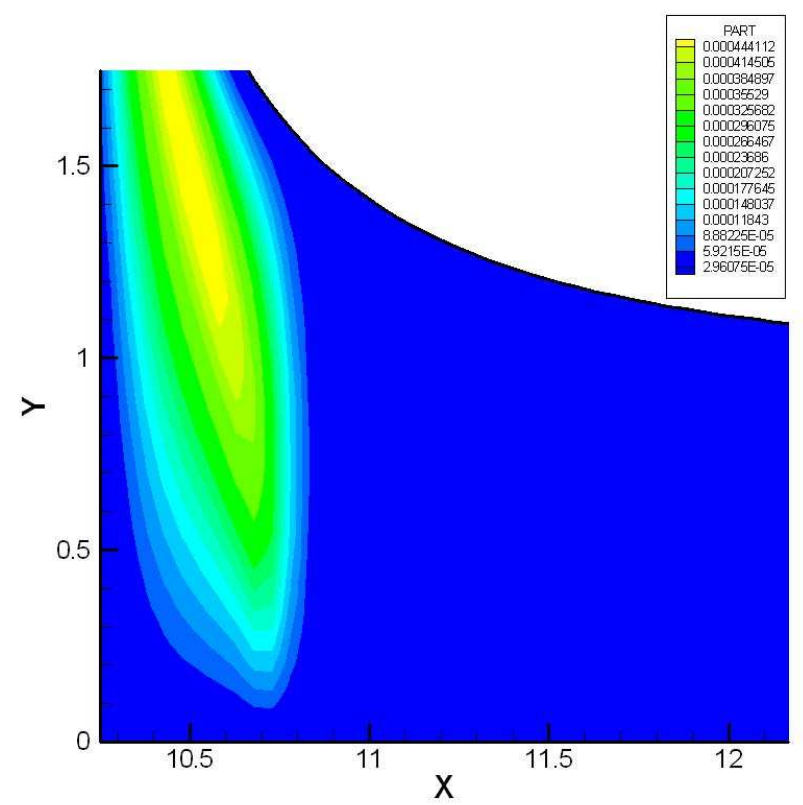

Figure 3: (Color online.) Particle concentration field in the near-wall layer; $m=30$. 


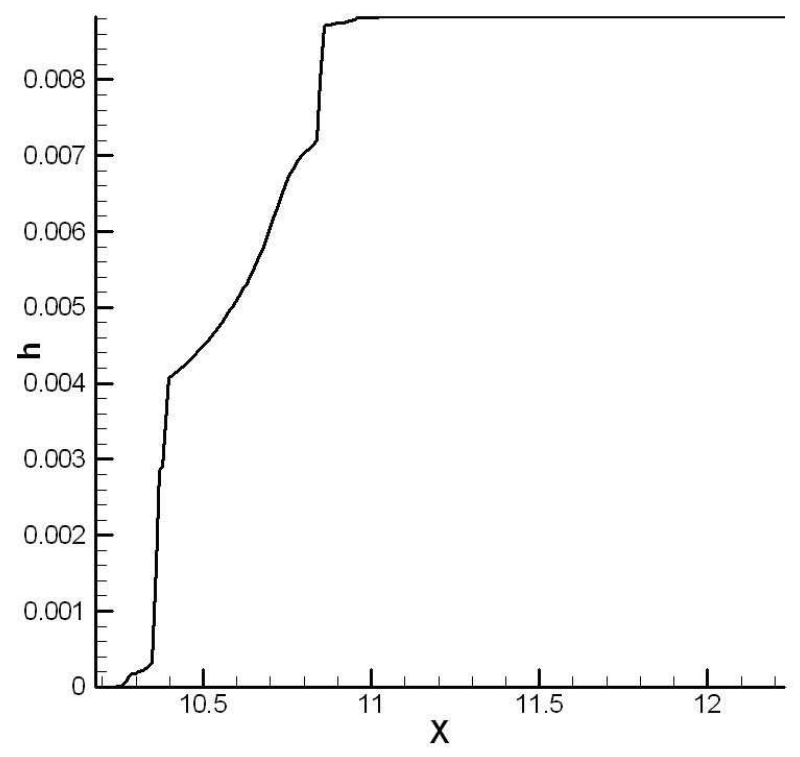

Figure 4: Deposited layer thickness along the wall; $m=30$.

as the deposited layer thickness also increases (cf. Figs. 4 and 6). Also, the deposited layer has a lesser roughness in the case of $\mathrm{m}=3$ (Fig. 6).

\section{Effect of swelling islands}

Consider a material with $\mathrm{n}$ potential defects per unit volume. These potential defects are normally closed but can be opened due to stretching in any direction if an appropriate local stress arises. Being completely open, a defect becomes a new nanopore. In electrospun polymer nanofibers such potential defects can represent possible nano-delamination sites and imperfections in macromolecular network. A typical content of PNIPAM in nanofibers is about $25 \%$ of the overall polymer mass. PNIPAM molecules could be either considered as defects themselves, or as the islands dispersed between the defects. In the first case, PNIPAM swelling will result in defect opening. In the second case, PNIPAM shrinkage in polymer matrix will result in stretching of the defects and opening of new nanopores. The latter scenario seems to be more plausible.

Consider a defect as a potential rupture surface, with its banks being initially conglutinated (cf. Fig. 7). The banks can be pulled apart by an appropriate stress normal to the surface. Moreover, each defect is actually a site of multiple conglutinated surfaces radiating spherically symmetrically, each of them could be opened by an appropriate normal stress. Then, a completely open defect (three intersecting shallow gaps; cf. Fig. 7) will make a single nanopore. Obviously, defects differ from each other due to many random factors in electrospinning. Therefore, the defect-opening process can be considered as random. The probability density function of a defect to be opened by 


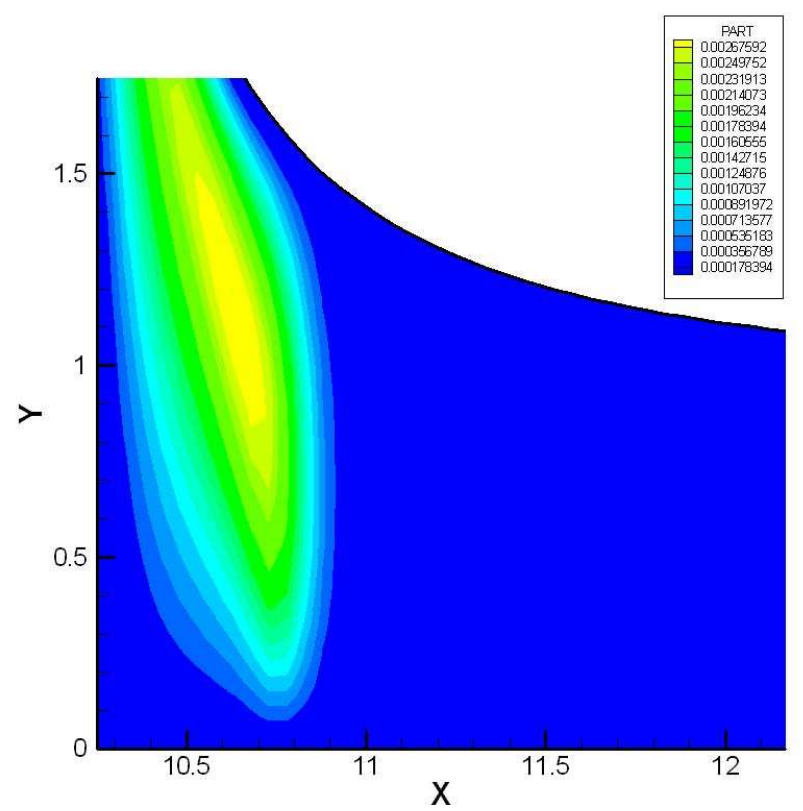

Figure 5: (Color online.) Particle concentration field in the near-wall layer; $m=3$.

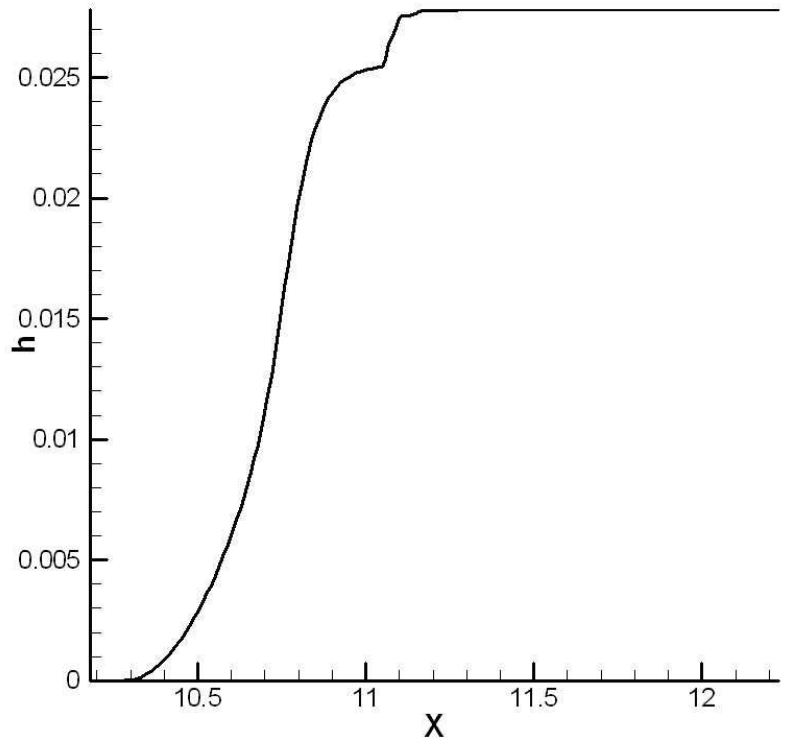

Figure 6: Deposited layer thickness along the wall; $m=3$. 


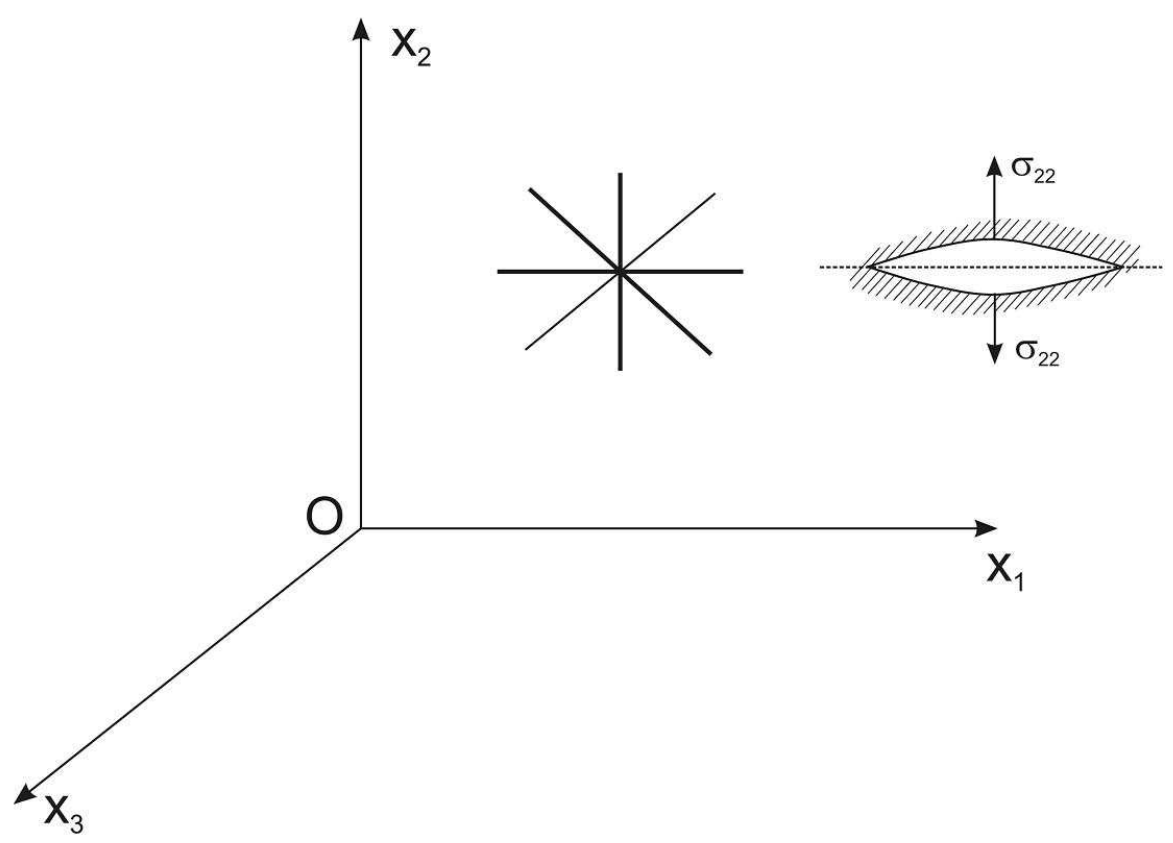

Figure 7: Sketch of a defect and its opening. The closed defect with conglutinated banks is shown on the left. A defect opened by stresses in $x_{2}$ direction is shown on the right.

a normal stress $\sigma_{11}$ (related to stretching along an axis $O x_{1}$, whereas the conglutinated surface is normal to this axis) is $F\left(\sigma_{11}\right)$, and the probability of the defect to be opened by a stress from the interval $\left[\sigma_{11}, \sigma_{11}+d \sigma_{11}\right]$ is $p_{1}=F\left(\sigma_{11}\right) d \sigma_{11}$. The defect-opening events in different directions are considered to be mutually independent. Therefore, the number of new nanopores in a unit volume subjected to stretching, for example, along three normal axes $O x_{1}, O x_{2}$, and $O x_{3}$ is

$$
d N=n F\left(\sigma_{11}\right) d \sigma_{11} F\left(\sigma_{22}\right) d \sigma_{22} F\left(\sigma_{33}\right) d \sigma_{33}
$$

On the other hand, $d N$ is obviously related to the joint probability density function $f\left(\sigma_{11}, \sigma_{22}, \sigma_{33}\right)$,so that

$$
d N=n F\left(\sigma_{11}\right) F\left(\sigma_{22}\right) F\left(\sigma_{33}\right) d \sigma_{11} d \sigma_{22} d \sigma_{33}=f\left(\sigma_{11}, \sigma_{22}, \sigma_{33}\right) d \sigma_{11} d \sigma_{22} d \sigma_{33} .
$$

Then, the number of defects in unit volume which will not be opened at all in such three-axial stretching with the corresponding stresses $\sigma_{1}, \sigma_{2}$, and $\sigma_{3}$ will be

$$
\begin{gathered}
N_{0}=n \int_{\sigma_{1}}^{\infty} F\left(\sigma_{11}\right) d \sigma_{11} \int_{\sigma_{2}}^{\infty} F\left(\sigma_{22}\right) d \sigma_{22} \int_{\sigma_{3}}^{\infty} F\left(\sigma_{33}\right) d \sigma_{33}=n \phi\left(\sigma_{1}\right) \phi\left(\sigma_{2}\right) \phi\left(\sigma_{3}\right)= \\
\int_{\sigma_{1}}^{\infty} \int_{\sigma_{2}}^{\infty} \int_{\sigma_{3}}^{\infty} f\left(\sigma_{11}, \sigma_{22}, \sigma_{33}\right) d \sigma_{11} d \sigma_{22} d \sigma_{33}=\omega\left(\sigma_{1}, \sigma_{2}, \sigma_{3}\right) .
\end{gathered}
$$

The number of defects opened in a unit volume in this case is $N=n-N_{0}$. 
The same situation can be obviously described in the framework of an arbitrarily oriented Cartesian coordinate frame $O x, O y$ and $O z$. Then, in fact the number of defects which weren't opened $N_{0}=\omega\left(\sigma_{x x}, \sigma_{x y}, \sigma_{x z}, \sigma_{y y}, \sigma_{y z}, \sigma_{z z}\right)$, where $\sigma_{x x}$, etc. denote the corresponding components of the stress tensor $\boldsymbol{\sigma}$. The previously used Cartesian axes $O x_{1}, O x_{2}$, and $O x_{3}$ are now considered as the principal axes of the stress tensor $\boldsymbol{\sigma}$, and $\sigma_{1}, \sigma_{2}$, and $\sigma_{3}$ become, correspondingly, the principal stresses. Obviously, the number of conglutinated or opened defects should not depend on the directions of the arbitrarily chosen axes $O x, O y$ and $O z$, which means that the function $\omega$ should depend only on the three invariants of the stress tensor $\sigma$ :

$$
\begin{gathered}
I_{1}=\sigma_{x x}+\sigma_{y y}+\sigma_{z z}=\sigma_{1}+\sigma_{2}+\sigma_{3}, \\
I_{2}=\sigma_{x x} \sigma_{y y}+\sigma_{y y} \sigma_{z z}+\sigma_{x x} \sigma_{z z}-\sigma_{x y}^{2}-\sigma_{y z}^{2}-\sigma_{x z}^{2}=\sigma_{1} \sigma_{2}+\sigma_{2} \sigma_{3}+\sigma_{1} \sigma_{3}, \\
I_{3}=\sigma_{x x} \sigma_{y y} \sigma_{z z}+2 \sigma_{x y} \sigma_{y z} \sigma_{x z}-\sigma_{x x} \sigma_{y z}^{2}-\sigma_{y y} \sigma_{x z}^{2}-\sigma_{z z} \sigma_{x y}^{2}=\sigma_{1} \sigma_{2} \sigma_{3} .
\end{gathered}
$$

Equations (4.3)-(4.6) yield the following functional equation:

$$
n \phi\left(\sigma_{1}\right) \phi\left(\sigma_{2}\right) \phi\left(\sigma_{3}\right)=\omega\left(\sigma_{1}+\sigma_{2}+\sigma_{3}, \sigma_{1} \sigma_{2}+\sigma_{2} \sigma_{3}+\sigma_{1} \sigma_{3}, \sigma_{1} \sigma_{2} \sigma_{3}\right) .
$$

Its solution reads

$$
\phi\left(\sigma_{i}\right)=\left(A+B \sigma_{i}\right) \exp \left(-C \sigma_{i}\right), \quad i=1,2,3,
$$

where $A, B$ and $C$ are constants.

If there is no stress applied, then the number of conglutinated defects in a unit volume $N_{0}=n$. On the other hand, in this case Eqs. (4.7) and (4.8) yield $N_{0}=n A^{3}$. Therefore, $A=1$.

Using Eq. (4.8), one finds

$$
\phi\left(\sigma_{i}\right)=\int_{\sigma_{i}}^{\infty} F\left(\sigma_{i i}\right) d \sigma_{i i}=\left(1+B \sigma_{i}\right) \exp \left(-C \sigma_{i}\right)
$$

which yields

$$
F\left(\sigma_{i i}\right)=\left(B C \sigma_{i i}+C-B\right) \exp \left(-C \sigma_{i i}\right) .
$$

Since material has a certain strength, $F(0)=0$, and thus $C=B$, which yields

$$
F\left(\sigma_{i i}\right)=B^{2} \sigma_{i i} \exp \left(-B \sigma_{i i}\right)
$$

which obviously satisfies the normalization condition.

The mathematical expectation of the defect-opening stress, $\sigma_{*}$, is in fact identical to the tensile strength of material, which is a known parameter $\left(\sigma_{*}\right.$ is of the order of $100 \mathrm{MPa}$ for electrospun nanofibers [26]). Therefore, using Eq. (4.11), one has

$$
\sigma_{*}=\int_{0}^{\infty} \sigma_{i i} F\left(\sigma_{i i}\right) d \sigma_{i i}=\int_{0}^{\infty} B^{2} \sigma_{i i}^{2} \exp \left(-B \sigma_{i i}\right) d \sigma_{i i}
$$


which yields $B=2 / \sigma_{*}$. Then, from Eq. (4.7) it is possible to find the number of defects which weren't opened in a unit volume:

$$
\begin{gathered}
N_{0}=n\left(1+\frac{2}{\sigma_{*}}\left(\sigma_{1}+\sigma_{2}+\sigma_{3}\right)+\frac{4}{\sigma_{*}^{2}}\left(\sigma_{1} \sigma_{2}+\sigma_{2} \sigma_{3}+\sigma_{1} \sigma_{3}\right)+\frac{8}{\sigma_{*}^{3}} \sigma_{1} \sigma_{2} \sigma_{3}\right) \times \\
\exp \left(-\frac{2}{\sigma_{*}}\left(\sigma_{1}+\sigma_{2}+\sigma_{3}\right)\right) .
\end{gathered}
$$

The number of new defects opened in a unit volume $N=n-N_{0}$ is

$$
\begin{gathered}
N_{0}=n-n\left(1+\frac{2}{\sigma_{*}}\left(\sigma_{1}+\sigma_{2}+\sigma_{3}\right)+\frac{4}{\sigma_{*}^{2}}\left(\sigma_{1} \sigma_{2}+\sigma_{2} \sigma_{3}+\sigma_{1} \sigma_{3}\right)+\frac{8}{\sigma_{*}^{3}} \sigma_{1} \sigma_{2} \sigma_{3}\right) \times \\
\exp \left(-\frac{2}{\sigma_{*}}\left(\sigma_{1}+\sigma_{2}+\sigma_{3}\right)\right) .
\end{gathered}
$$

Assume that the dispersed PNIPAM islands inside a matrix are affected by some stimuli which trigger their swelling or shrinkage. Denote the relative volume change due to spherically symmetric volume swelling/shrinkage related to the islands as $\mathrm{m}$. Assume that the material obeys Hooke's law. Then, the magnitudes of the radial, and two azimuthal normal stresses represent themselves the principal stresses and thus

$$
\sigma_{1}=\sigma_{2}=\sigma_{3}=\frac{E}{3\left(1-2 \nu_{P}\right)}|m|,
$$

where $E$ is Young's modulus (which is of the order of $E=1 \mathrm{GPa}$ for electrospun nanofibers [2, 26]) and $\nu_{P}$ Poisson's ratio.

Denote these equal principal stresses as $\sigma_{p}$. Then, substituting Eq. (4.15) in (4.14), and considering the limit $\sigma_{p} / \sigma_{*}<<1$, we find the number of new nanopores in a unit volume as

$$
N=6 n \frac{\sigma_{p}^{2}}{\sigma_{*}^{2}}=\frac{2}{3} n\left(\frac{E}{\sigma_{*}}\right)^{2} \frac{1}{\left(1-2 \nu_{P}\right)^{2}} m^{2} .
$$

The number of new nanopores with openings per unit surface area of such a material is proportional to $N^{2 / 3}$. According to [9], release rates in desorption-limited processes are governed by two parameters, only one of which (the nanoporosity factor $\alpha$ ) is affected by the number of nanopores with openings at the fiber surface. Therefore,

$$
\alpha-\alpha_{0} \sim\left(\frac{2}{3}\right)^{2 / 3} n^{2 / 3}\left(\frac{E}{\sigma_{*}}\right)^{4 / 3} \frac{1}{\left(1-2 \nu_{P}\right)^{4 / 3}}|m|^{4 / 3},
$$

where $\alpha_{0}$ is the nanoporosity factor in a composite nanofiber before PNIPAM molecules have changed their conformation.

In addition, we assume that the islands do not affect the desorption enthalpy of the matrix. Since $|m| \sim C$, where $C$ denotes the island concentration, one finds from Eq. (4.17) 


$$
\alpha-\alpha_{0} \sim\left(\frac{2}{3}\right)^{2 / 3} n^{2 / 3}\left(\frac{E}{\sigma_{*}}\right)^{4 / 3} \frac{1}{\left(1-2 \nu_{P}\right)^{4 / 3}} C^{4 / 3} .
$$

The analysis of dye release data allows measurement of the nanoporosity factor [9]. Therefore, the dependence (4.18) (in particular, the fact that $\alpha-\alpha_{0} \sim C^{4 / 3}$ ) can be verified or disapproved by the experimental data. In addition, it is emphasized that the factor $\left(E / \sigma_{*}\right)^{4 / 3} /\left(1-2 \nu_{P}\right)^{4 / 3}$ in Eq. (4.18) can be of the order of 10-100, and thus the effect of even a minute concentration of the island material on the release rate can be significant.

\section{Conclusion}

Electrospun nanofiber-based composites incorporating stimuli-responsive guest polymers (e.g., PNIPAM) represent a novel field of nanotechnology. Mathematical theory of their production and activation is in its infancy. The present work outlines two possible modeling directions leading to certain predictions, which call for further experimental verification and theoretical extensions.

\section{Acknowledgement}

This material is based upon work supported by the National Science Foundation under Grants NIRT CTS-0609062 and CBET-0543538.

\section{References}

[1] D.H. Reneker, A.L. Yarin, E. Zussman, H. Xu. Electrospinning of nanofibers from polymer solutions and melts. Advances in Applied Mechanics, 41 (2007), 43-195.

[2] A.L. Yarin, E. Zussman, J.H. Wendorff, A. Greiner. Material encapsulation in core-shell micro/nanofibers, polymer and carbon nanotubes and micro/nanochannels. J. Mater. Chem., 17 (2007), 2585-2599.

[3] H. Chen, Y.L. Hsieh. Ultrafine hydrogel fibers with dual temperature- and $p H$ - responsive swelling behaviors. J. Polym. Sci.: Part A: Polymer Chemistry, 42 (2004), 6331-6339.

[4] A. Chunder, S. Sarkar, Y. Yu, L. Zhai. Fabrication of ultrathin polyelectrolyte fibers and their controlled release properties. Colloids and Surfaces B: Biointerfaces, 58 (2007), 172-179.

[5] J. Ye, Y. Hou, G. Zhang, C. Wu. Temperature-induced aggregation of Poly ( $N$ isopropylacrylamide)-stabilized CdS quantum dots in water. Langmuir, 24 (2008), 27272731.

[6] D. Liang, B.S. Hsiao, B. Chu. Functional electrospun nanofibrous scaffolds for biomedical applications. Advanced Drug Delivery Reviews, 59 (2007), 1392-1412. 
[7] J. Kim, R.G. Carbonell. Deposition of small organic molecules by the displacement of two immiscible supercritical phases. Langmuir, 22 (2006), 2117-2129.

[8] J. Kim, B.J. Novick, J.M. DeSimone, R.G. Carbonell. Ultrathin film deposition by liquid $\mathrm{CO}_{2}$ free meniscus coating-uniformity and morphology. Langmuir, 22 (2006), 642-657.

[9] R. Srikar, A.L. Yarin, C.M. Megaridis, A.V. Bazilevsky, E. Kelley. Desorption-limited mechanism of release from polymer nanofibers. Langmuir, 24 (2008), 965-974.

[10] L.D. Landau, V.G. Levich. Dragging of a liquid by a moving plate. Acta Physicochimica USSR, 17 (1942), 42-54.

[11] V.G. Levich. Physicochemical hydrodynamics. Prentice-Hall, Englewood Cliffs, 1962.

[12] H. Schlichting. Boundary layer theory. McGrow-Hill, New York, 1979.

[13] Ya. B. Zeldovich. On the theory of new phase formation: cavitation. JETP, 12 (1942), 525528.

[14] Ya. B. Zeldovich. On the theory of new phase formation: cavitation. Acta Physicochimica USSR, 18 (1943), 1-22.

[15] Ya. B. Zeldovich. On the theory of new phase formation: cavitation. In Selected Works of Ya.B. Zeldovich, Vol. 1, Chemical Physics and Hydrodynamics. Princeton University Press, Princeton, 1992.

[16] I.V. Markov. Crystal growth for beginners. World Scientific, River Edge, 2003.

[17] M.A. Lavrentiev, V.B. Shabat. Hydrodynamic problems and their mathematical models. Nauka, Moscow, 1973 (in Russian).

[18] K.E. Dzhaugashtin, A.L. Yarin. Numerical simulation of non-self-similar wall jet. J. Engineering Physics, 32 (1977), 420-426.

[19] K.E. Dzhaugashtin, A.L. Yarin. Combustion process in laminar homogeneous gas jets. Combustion, Explosion and Shock Waves, 14 (1978), 321-327.

[20] K.E. Dzhaugashtin, A.L. Yarin. Combustion in laminar diffusion gas jets. Combustion, Explosion and Shock Waves, 15 (1979), 92-95.

[21] K.E. Dzhaugashtin, A.L. Yarin. Grazing flame core of unmixed gases. Combustion, Explosion and Shock Waves, 17 (1981), 283-289.

[22] E. Bekturganov, K.E. Dzhaugashtin, Z.B. Sakipov, A.L. Yarin. Jet flow over a moving wall. Fluid Mechanics - Soviet Research, 11 (1982), 14-24.

[23] A.L. Yarin. Aerodynamics of a gas flame serving as a source of solid particles. Combustion, Explosion and Shock Waves, 20 (1984), 686-689. 
[24] M.T. Murzabayev, A.L. Yarin. Dynamics of sprinkler jets. Fluid Dynamics, 20 (1985), 715722.

[25] A.L. Yarin, G. Brenn, J. Keller, M. Pfaffenlehner, E. Ryssel, C. Tropea. Flow field characteristics of an aerodynamic acoustic levitator. Phys. Fluids, 9 (1997), 3300-3314.

[26] E. Zussman, M. Burman, A.L. Yarin, R.Khalfin, Y. Cohen. Tensile deformation of electrospun Nylon 6,6 nanofibers. J. Polym. Sci., Part B - Polymer Physics, 44 (2006), 1482-1489. 